\title{
Automated Dysarthria Severity Classification for Improved Objective Intelligibility Assessment of Spastic Dysarthric Speech
}

\author{
Milton Sarria Paja and Tiago H. Falk \\ Institut National de la Recherche Scientifique (INRS-EMT) \\ University of Quebec, Montreal, QC, Canada
}

\begin{abstract}
In this paper, automatic dysarthria severity classification is explored as a tool to advance objective intelligibility prediction of spastic dysarthric speech. A Mahalanobis distance-based discriminant analysis classifier is developed based on a set of acoustic features formerly proposed for intelligibility prediction and voice pathology assessment. Feature selection is used to sift salient features for both the disorder severity classification and intelligibility prediction tasks. Experimental results show that a two-level severity classifier combined with a 9-dimensional intelligibility prediction mapping can achieve 0.92 correlation and 12.52 root-mean-square error with subjective intelligibility ratings. The effects of classification errors on intelligibility accuracy are also explored and shown to be insignificant.
\end{abstract}

Index Terms: Intelligibility, dysarthria, diagnosis.

\section{Introduction}

Discriminating healthy and pathological speech patterns is essential to the clinical diagnosis of speech disorders, which is usually carried out by e.g., speech-language pathologists and vocologists. Moreover, characterizing the effects of a particular speech disorder on intelligibility is also crucial, as it helps identify disorder severity, guides treatments and interventions, as well as documents improvements over time. Commonly, such analyses are performed via subjective listening tests, which are costly, laborious, and prone to examiner internal biases (e.g., knowledge of the patient and/or their disorder). Automatic acoustical analysis for classification and intelligibility prediction, on the other hand, has several advantages, such as repeatability, cost and labour reduction, and it opens doors for remote patient rehabilitation. Ongoing efforts have focused on objective disorder classification and intelligibility estimation (e.g., [1, 2])

Recently, an intelligibility prediction algorithm was developed for spastic dysarthric speech [2]. Spastic dysarthria is one of the most common types of speech dysarthrias and can be associated with various aetiologies, including cerebral palsy and traumatic brain injury [3]. Spastic dysarthria is characterized by excessive

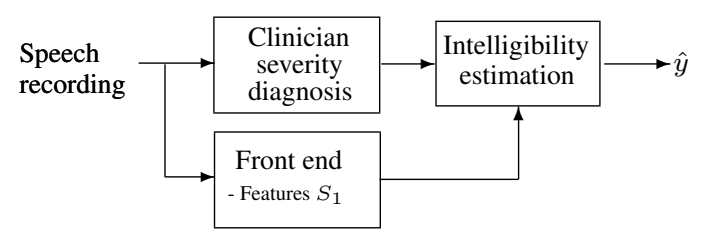

Figure 1: Block diagram of objective dysarthric speech intelligibility algorithm described in [2].

nasalization, disordered speech prosody, imprecise articulation, and variable speech rate which often render the speech unintelligible [3]. In order to accurately characterize spastic dysarthria intelligibility, a multidimensional approach was required to account for each of the abovementioned perceptual dimensions [2].

As has been previously reported, however, speech impairments may differ not only with dysarthria type, but also by the severity of the disorder [4]. As an example, low pitch variation (monotonicity) has been associated with mild dysarthria, whereas high pitch variation has been associated with speakers with severe disorders $[4,2]$. As such, in order to develop reliable speech intelligibility predictors, different mappings need to be devised between the extracted acoustic features and an intelligibility rating, based on the severity of the disorder. In [2], the authors used human intervention in order to classify speech samples into mid-low and mid-high intelligibility classes (a direct measure of dysarthria severity), prior to using a "class-based" intelligibility predictor (see Fig. 1); performance gains of $8 \%$ were achieved relative to using a "global" mapping.

Ultimately, it is expected that an intelligibility assessment tool will operate without the need for human intervention, particularly in remote rehabilitation applications. In such scenarios, an automated dysarthria classification tool is needed. This paper describes one such tool and presents an improved intelligibility prediction algorithm for spastic dysarthric speech. The two-stage algorithm makes use of previously-proposed features [2] plus features which have been previously used in voice pathology assessment; only salient features are used. The effects of classification errors on intelligibility prediction 
accuracy is also assessed and shown to be insignificant.

\section{Proposed spastic dysarthria severity classification and intelligibility prediction}

\subsection{Previously-explored acoustic features}

In [2], several acoustic features were extracted from speech signals in order for perturbations across multiple perceptual dimensions to be characterized. More specifically, features were extracted to characterize atypical vocal source excitation, temporal dynamics, nasality, and prosody. A subset of six of these features were shown to be useful for speech intelligibility prediction. While a complete description of the six features is beyond the scope of this paper (the interested reader is referred to [2] for more details), a quick description is given here for the sake of completeness.

First, in order to characterize atypical vocal source excitation (related to vocal harshness, breathiness), the kurtosis of the linear prediction $(\mathrm{LP})$ residual $\left(\mathcal{K}_{L P}\right)$ signal was used. Commonly, the residual signal characterizes the vocal source excitation. The LP residual signal of healthy clean speech is characterized by strong impulselike peaks associated with glottal pulses. For pathological speech, more noise-like excitation patterns are observed, which in turn cause a decrease in the LP residual kurtosis. Second, in order to characterize speech temporal impairments (e.g., unclear distinction between adjacent phonemes due to imprecise placement of articulators), two features were developed, one focusing on short-term (60 ms) temporal dynamics and the other on long-term (256 ms) dynamics. The former was characterized by the rate-of-change of the signal log-energy, computed as the standard deviation of the delta zeroth order cepstral coefficient $\left(\sigma_{\Delta c_{0}}\right)$. The latter, in turn, made use of an auditory-inspired modulation spectral signal representation [5] and represented the ratio of modulation spectral energy at modulation frequencies lower than $4 \mathrm{~Hz}$ to modulation frequencies greater than $4 \mathrm{~Hz}$; the feature is termed low-to-high modulation energy ratio (LHMR).

Lastly, while nasality related parameters did not contribute to the task at hand, three prosody-related features stood out, namely the standard deviation of the fundamental frequency $\left(\sigma_{f_{0}}\right)$, the range of $f_{0}\left(\Delta_{f_{0}}\right)$, and percentage of voiced segments in the uttered word $(\% \mathcal{V})$. For notation purposes, these six features are grouped into a feature set termed $S_{1}$ and given by:

$$
S_{1}=\left\{\mathcal{K}_{L P}, \sigma_{\Delta c_{0}}, \text { LHMR, } \sigma_{f_{0}}, \Delta_{f_{0}}, \% \mathcal{V}\right\}
$$

\subsection{Alternate complementary features}

While the feature set $S_{1}$ described above proved useful for automated intelligibility prediction, the six proposed features may not be optimal for automated disorder severity classification. To this end, we explore the inclusion of alternate features which have been explored for voice pathologies. More specifically, we target three new representations, namely mel frequency cepstral coefficients (MFCCs), glottal-to-noise excitation ratio (GNE), and harmonics-to-noise ratio (HNR). MFCCs were considered here due to their capability of capturing irregular vocal fold movements or the lack of vocal-fold closure due to mass/tissue changes [6]; 11th order MFCCs were used in the experiments described herein.

The GNE and HNR features, in turn, were introduced for their capability of measuring the degree of "noise" generated by the disorders. GNE quantifies the ratio of excitation due to vocal fold oscillations versus the excitation given by turbulent noise [7]. The feature is closely related to breathiness but somewhat complementary to the $\mathcal{K}_{L P}$ feature described above. Lastly, HNR measures the ratio between the energy of periodic signal components to the energy of aperiodic signal components. It can be seen as a signal-to-aspiration noise ratio when other aperiodicities in the signal are comparatively low [8]. The three abovementioned feature representations are computed on a per-window basis (40ms Hamming windows, $50 \%$ overlap). For the task at hand, four statistical values are computed over the duration of the speech signal: mean, standard deviation, skewness, and kurtosis. In the case of the MFCC feature representation, the four statistical values are computed for each of the 11 coefficients. For notation purposes, the 52 new acoustic feature set $(4 \times 11 \mathrm{MFCCs}+4 \times \mathrm{GNE}+4 \times \mathrm{HNR})$ is termed $S_{2}$.

\subsection{Proposed system}

Figure 2 depicts the block diagram of the proposed dysarthric speech intelligibility prediction system. It builds on the previously-developed algorithm (see Fig. 1) by replacing the clinician-dependent severity classification block by one that is automated. A larger pool of acoustic features are extracted $\left(S_{1} \cup S_{2}\right)$ and salient features are found for both the classification and prediction tasks at hand. For salient feature selection, a correlation-based sequential feature selection algorithm is used. For intelligibility estimation, a composite linear mapping function $g(\cdot)$ is used to estimate intelligibility ratings $(\hat{y})$ from $N$ features $f_{i}$, i.e.:

$$
\hat{y}=g(\mathbf{f})=A_{0}+\sum_{i=1}^{N} A_{i} f_{i} .
$$

With the proposed system, two mapping functions are used, namely $g_{m i d-l o w}$ and $g_{m i d-h i g h}$, to predict intelligibility scores of moderate-to-severe (mid-to-low intelligibility levels) and moderate-to-mild (mid-to-high intelligibility levels) dysarthric speakers, respectively. Having said this, the developed classifiers have been trained to discriminate between these two severity classes. 


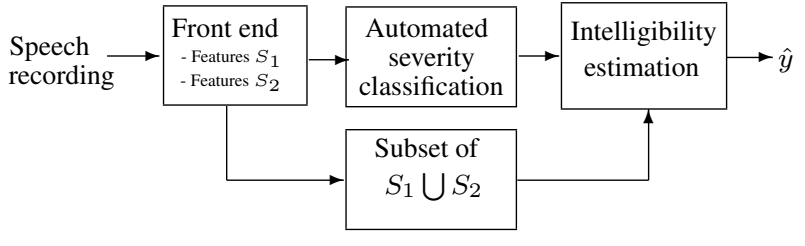

Figure 2: Block diagram of the proposed combined severity classiifcation and intelligibility estimation system.

\section{Experimental Setup}

The data used in our experiments consisted of the audio content of the Universal Access (UA-Speech) audiovisual database made publicly-available by the University of Illinois and described in detail in [9]. The dataset was originally recorded using a 7-channel microphone array; here, data recorded from the centre microphone was used, as in [2]. Data from 10 spastic dysarthric speakers (3 female) were used and covered a wide range of disorder severity, ranging from $2 \%$ to $95 \%$ word intelligibility. Each speaker read 765 isolated word utterances including the 10 digits (zero to nine), 26 radio alphabet letters (e.g., Alpha, Bravo), 19 computer commands (e.g., backspace, delete), the 100 most common words in the Brown corpus of written English (e.g., it, is, you), and 300 uncommon words selected from children's novels [9].

To evaluate the discriminative capabilities of the different features, three different discriminant analysis classifiers were explored based on linear, quadratic and Mahalanobis-distance discriminant functions. Randomized bootstrap (15-fold) cross validation was used with $70 \%$ of the input data recordings kept for system training and $30 \%$ left for validation. Classification accuracy is reported as average accuracy \pm the standard error across the 15 cross-validation trials [10]. Moreover, in order to explore the effects of dysarthria severity misclassification on intelligibility prediction accuracy, two scenarios were investigated. The first, considered the gold standard for the task at hand, assumes perfect classification, akin to what would be achieved with an experienced clinician (scenario \#1). The second represents a more practical system which uses the trained classifier to select from the possible $g_{\text {mid-low }}$ or $g_{\text {mid-high }}$ mapping functions (scenario \#2). Two system performance metrics were used, namely the root-mean-square error and the Pearson correlation coefficient computed between the true subjective intelligibility ratings and the estimated scores.

\section{Experimental results}

\subsection{Dysarthria severity classification}

Table 1 reports the classification accuracy obtained with different feature set combinations and classifiers. More
Table 1: Classification accuracy using different feature combinations and classifiers.

\begin{tabular}{|c|c|c|c|}
\hline \multirow{2}{*}{$\begin{array}{c}\text { feature } \\
\text { set }\end{array}$} & \multicolumn{3}{|c|}{ Classifier } \\
\cline { 2 - 4 } & Linear & Quadratic & Mahalanobis \\
\hline $\mathrm{S}_{1}$ & $83.9 \pm 0.04$ & $82.5 \pm 0.03$ & $83.5 \pm 0.05$ \\
\hline $\mathrm{S}_{2}$ & $87.9 \pm 0.04$ & $93.0 \pm 0.03$ & $92.4 \pm 0.03$ \\
\hline $\mathrm{FS}\left\{\mathrm{S}_{2}\right\}$ & $86.2 \pm 0.03$ & $92.5 \pm 0.04$ & $92.5 \pm 0.03$ \\
\hline $\mathrm{FS}\left\{\mathrm{S}_{2}\right\}+\mathrm{S}_{1}$ & $91.8 \pm 0.03$ & $94.3 \pm 0.02$ & $94.9 \pm 0.03$ \\
\hline
\end{tabular}

specifically, the following feature set combinations were explored: $S_{1}$ alone, $S_{2}$ alone, the 28 most salient $S_{2}$ features (represented as 'FS $\left\{S_{2}\right\}$ '), and a combination of $S_{1}$ and FS $\left\{S_{2}\right\}$. As can be seen, for $S_{1}$, all three classifiers resulted in similar accuracy. For all other feature combinations, quadratic and Mahalanobis-distance based classifiers resulted in improved performance. Overall, the Mahalanobis distance classifier with the $\operatorname{FS}\left\{S_{2}\right\}+S_{1}$ feature set resulted in the best accuracy $(95 \%)$.

\subsection{Dysarthric speech intelligibility prediction}

In order to develop the composite mappings $g_{\text {mid-low }}$ and $g_{m i d-h i g h}$, the UA-Speech database was partitioned into two disjoint sets. Speech files belonging to the "uncommon word" category (300 files per participant) served as unseen test data and the remaining files (465 files per participant) served as training data and were used to i) select salient features for intelligibility prediction and ii) to obtain the weights $A i$ for the mapping functions (1). As with the classifier, sequential forward feature selection was used to sift the top features for intelligibility prediction. Table 2 shows the top- 9 features selected for prediction along with their correlations (R) with the subjective intelligibility rating. As can be seen, five of the nine features are those previously proposed in [2] and four are complementary ones explored here. The new feature set with these nine features is represented as 'FS $\left\{S_{1} \bigcup S_{2}\right\}$ '. Table 3 shows the weights $A_{i}$ from (Eq. 1) obtained for the $g_{\text {mid-low }}$ and $g_{\text {mid-high }}$ mappings. The difference in weight values between the two mappings corroborates previous findings showing that speech impairments also differ by severity level $[2,4]$.

Lastly, in order to investigate the potential detrimental effects of severity level misclassification on intelligibility prediction, Table 4 shows the correlation $(\mathrm{R})$ and rootmean-square error $\left(\varepsilon_{r m s}\right)$ between subjective and predicted intelligibility ratings in scenarios \#1 and \#2, as described in Section 3. For scenario \#2, the Mahalanobisbased classifier trained on the FS $\left\{S_{2}\right\}+S_{1}$ feature set was used. As can be seen, by using the feature set $S_{1}$ proposed in [2], the 5\% classification error seen in Table 1 resulted in a 3\% decrease in $\mathrm{R}$ and a $7 \%$ increase in $\varepsilon_{r m s}$ relative to the gold standard. With the updated 
Table 2: Nine most salient features for the intelligibility prediction task. Column ' $\mathrm{R}$ ' represent the correlation between the feature and the subjective intelligibility rating

\begin{tabular}{|l|r|}
\hline feature & \multicolumn{1}{|c|}{$\mathbf{R}$} \\
\hline \hline$\sigma_{c_{2}}$ & 0.90 \\
$\mathcal{K}_{L P}$ & 0.89 \\
$\% \mathcal{V}$ & -0.83 \\
$\sigma_{\Delta c_{0}}$ & 0.79 \\
$\bar{c}_{4}$ & 0.75 \\
$\Delta_{f_{0}}$ & -0.72 \\
$\mathrm{LHMR}$ & 0.69 \\
$\sigma_{c_{1}}$ & 0.67 \\
$\sigma_{c_{11}}$ & -0.66 \\
\hline
\end{tabular}

Table 3: Weights $A_{i}$ of the two per-severity intelligibility prediction mappings given by (Eq. 1)

\begin{tabular}{|l|r|r|}
\hline \multicolumn{1}{|c|}{ Weight } & $g_{\text {mid-low }}$ & $g_{\text {mid-high }}$ \\
\hline \hline$A_{0}$ & 18.94 & 67.53 \\
$A_{1}\left(\sigma_{c_{2}}\right)$ & 2.64 & 1.83 \\
$A_{2}\left(\mathcal{K}_{L P}\right)$ & 1.41 & 3.60 \\
$A_{3}(\% \mathcal{V})$ & -0.38 & -11.56 \\
$A_{4}\left(\sigma_{\Delta c_{0}}\right)$ & 1.82 & 2.42 \\
$A_{5}\left(\bar{c}_{4}\right)$ & -0.31 & -2.92 \\
$A_{6}\left(\Delta_{f_{0}}\right)$ & -0.02 & -2.21 \\
$A_{7}(\mathrm{LHMR})$ & 0.02 & -1.63 \\
$A_{8}\left(\sigma_{c_{1}}\right)$ & 3.77 & 0.94 \\
$A_{9}\left(\sigma_{c_{11}}\right)$ & -1.17 & -5.16 \\
\hline
\end{tabular}

feature set proposed here (see Table 2), the 5\% classification error resulted in a $4 \%$ decrease in $\mathrm{R}$ and a $4 \%$ increase in $\varepsilon_{r m s}$. These insignificant drops in performance are outweighed by the benefits of having an automated system that does not require human intervention. Moreover, Table 4 shows that the updated proposed feature set $\mathrm{FS}\left\{S_{1} \cup S_{2}\right\}$ reduces $\varepsilon_{r m s}$ by $11 \%$ and $13 \%$ relative to feature set $S_{1}$ in scenarios \#1 and \#2, respectively.

\section{Conclusions}

The design of a two-stage scheme for spastic dysarthria severity classification and intelligibility prediction is presented. First, different feature combinations and classifier types were explored for two-level dysarthria severity classification (i.e., mid-to-low and mid-to-high intelligibility levels). Experimental results showed that a Mahalanobis distance-based discriminant analysis classifier trained on a pool of 34 acoustic features could achieve 95\% classification accuracy. Second, a subset of nine salient features were used to train two intelligibility prediction mappings, one for mid-to-low intelligibility levels
Table 4: Performance comparison under two experimental scenarios (see Section 3 for more details)

\begin{tabular}{|c|c|c|c|c|}
\hline \multirow{2}{*}{$\begin{array}{c}\text { feature } \\
\text { set }\end{array}$} & \multicolumn{2}{|c|}{ scenario 1 } & \multicolumn{2}{c|}{ scenario 2 } \\
\cline { 2 - 5 } & $\mathrm{R}$ & $\varepsilon_{r m s}$ & $\mathrm{R}$ & $\varepsilon_{r m s}$ \\
\hline \hline$S_{1}$ & 0.95 & 13.43 & 0.92 & 14.41 \\
\hline $\mathrm{FS}\left\{S_{1} \cup S_{2}\right\}$ & 0.96 & 11.96 & 0.92 & 12.52 \\
\hline
\end{tabular}

and another for mid-to-high levels. When combined, improvements of up to $13 \%$ in intelligibility prediction accuracy could be achieved. Lastly, the effects of severity classification errors on intelligibility prediction accuracy was explored and found to be insignificant.

\section{Acknowledgements}

The authors wish to acknowledge Dr. Mark HasegawaJohnson for making the UA-Speech database available, and the Natural Sciences and Engineering Research Council of Canada for their financial support.

\section{References}

[1] M. Sarria-Paja and G. Castellanos-Domínguez, "Robust pathological voice detection based on component information from hmm," Advances in Nonlinear Speech Processing, pp. 254-261, 2011.

[2] T. H. Falk, W.-Y. Chan, and F. Shein, "Characterization of atypical vocal source excitation, temporal dynamics and prosody for objective measurement of dysarthric word intelligibility," Speech Communication, vol. 54, no. 5, pp. 622 - 631, 2012.

[3] P. Doyle, H. Leeper, A. Kotler, N. Thomas-Stonell, C. O'Neill, M. Dylke, and K. Rolls, "Dysarthric speech: a comparison of computerized speech recognition and listener intelligibility.," $J$ Rehabil Res Dev, vol. 34, no. 3, pp. 309-16, 1997.

[4] K. Schlenck, R. Bettrich, and K. Willmes, "Aspects of disturbed prosody in dysarthria," Clinical linguistics \& phonetics, vol. 7, no. 2, pp. 119-128, 1993.

[5] T. Falk and W.-Y. Chan, "Temporal dynamics for blind measurement of room acoustical parameters," Instrumentation and Measurement, IEEE Transactions on, vol. 59, pp. 978 -989, april 2010.

[6] J. I. Godino-Llorente, P. Gómez-Vilda, and M. Blanco-Velasco, "Dimensionality reduction of a pathological voice quality assessment system based on gaussian mixture models and short-term cepstral parameters.," IEEE Transactions on Biomedical Engineering, vol. 53, no. 10, pp. 1943-1953, 2006.

[7] J. I. Godino-Llorente, V. Osma-Ruiz, N. Saenz-Lechon, P. Gomez-Vilda, M. Blanco-Velasco, and F. Cruz-Roldan, "The effectiveness of the glottal to noise excitation ratio for the screening of voice disorders," Journal of voice : official journal of the Voice Foundation, vol. 24, pp. 47-56, 2010.

[8] P. J. Murphy and O. O. Akande, "Noise estimation in voice signals using short-term cepstral analysis," Journal of the Acoustical Society of America, vol. 121, no. 3, pp. 1679-1690, 2007.

[9] H. Kim, M. Hasegawa-Johnson, A. Perlman, J. Gunderson, T. S. Huang, K. Watkin, and S. Frame, "Dysarthric speech database for universal access research.," in INTERSPEECH, pp. 1741-1744, ISCA, 2008.

[10] D. G. Altman and J. M. Bland, "Standard deviations and standard errors.," BMJ British Medical Journal, vol. 331, no. 7521, p. 903 , 2005. 